\title{
The influence of ambient lighting on the detection of small contrast elements in digital dental radiographs
}

\author{
Till Schriewer • Ralf Schulze • Andreas Filippi • \\ Irene Mischak • Michael Payer • \\ Dorothea Dagassan-Berndt • Sebastian Kühl
}

Received: 12 July 2012 / Accepted: 1 October 2012 / Published online: 7 October 2012

(C) Springer-Verlag Berlin Heidelberg 2012

\begin{abstract}
Objectives The aim of this study was to evaluate the effect of ambient light on the detection of contrast elements in digital dental radiographs.

Materials and methods A high-contrast standardized digital radiograph of an aluminum step wedge containing 32 boreholes of different depth was cut into 40 isometric images. Images were presented at random on a 17-in cathode ray tube (CRT) monitor at different ambient background illuminations of 0, 50, 200, and $500 \mathrm{~lx}$. Twenty observers stated twice their blinded decision whether or not they could perceive a dark spot on a five-point confidence scale. Areas (Az) under receiver operating characteristic curves were calculated and compared between the four different ambient illuminations using the Friedman test. Statistical significance was set at $p<$ 0.05 . Overall agreement was estimated determining the intraclass correlation coefficient.
\end{abstract}

T. Schriewer $\cdot$ A. Filippi $\cdot$ D. Dagassan-Berndt $\cdot$ S. Kühl $(\bowtie)$ Department of Oral Surgery, Oral Radiology and Oral Medicine, School of Dental Medicine, University of Basel,

Hebelstrasse 3,

4056 Basel, Switzerland

e-mail: sebastian.kuehl@unibas.ch

\section{R. Schulze}

Department of Oral Surgery (and Oral Radiology),

School of Dental Medicine, University Medical Center

of the Johannes Gutenberg University Mainz,

Augustusplatz 2,

55131 Mainz, Germany

I. Mischak $\cdot$ M. Payer

Department of Oral Surgery and Radiology, School of Dentistry,

Medical University of Graz, Austria,

Auenbruggerplatz 12

8063 Graz, Austria
Results The Az values ( 0.735 for 0 lx, 0.728 for 50 lx, 0.735 for $200 \mathrm{~lx}$, and 0.788 for $500 \mathrm{~lx}$ ) did not significantly differ ( $p=0.796)$ between the four ambient lighting levels.

Conclusions The detection of small contrast features in digital dental radiographs on a CRT monitor seems to be comparable over a wide range of ambient background illumination. Clinical relevance The ambient light in dental offices of $<500$ lx may not negatively impact the diagnostic performance of digital dental radiographs.

Keywords Radiography · Digital images · Image perception $\cdot$ Visual perception $\cdot$ Light conditions

\section{Introduction}

Digital radiography has gained importance in daily clinical practice during the last decade [1]. When compared to conventionally film-based radiography, digital imaging reveals several advantages such as time economy, lower radiation dose, and the possibility of image processing after acquisition [2]. This, however, is associated with completely different viewing conditions. Conventional radiographs are mainly viewed in darkened rooms to optimize or maintain the perceived contrast of film images [3]. One known problem when evaluating conventional radiographs in darkened reading rooms is related to the extreme differences in illumination caused by the low ambient surrounding light and the high luminance image [3]. These differences may lead to advanced fatigue since the radiologist's pupils contract and dilate as the visual focus intermittently shifts between the high luminance monitor and the darker background wall, thus negatively affecting the diagnostic performance $[4,5]$.

Digital images in contrast are viewed on cathode ray tube (CRT) or liquid crystal display (LCD). One positive side 
effect of these different viewing conditions represents the lower diffuse reflection coefficient which allows a higher flexibility in adjusting the ambient light when compared to conventional viewing [4]. With regard to this, the background illumination may be elevated without negative influence on diagnostic performance. An elevated background illumination could positively influence the fatigue process during image evaluation. Modern dental offices are increasingly provided with monitors located in the direct surrounding of the dentist's chair. The background illumination in this surrounding is mainly elevated since light is needed for treatment purposes and additionally to create a friendly atmosphere. It could be shown that high levels of ambient light may decrease diagnostic accuracy by as much as $11 \%$ [6]. In contrast to this, others found a slight background illumination to improve the diagnostic performance with a positive effect on the sensitivity for digital images when compared to darkened conditions [3].

Nevertheless, there is a lack of information on the influence of ambient illumination on the diagnostic performance for digital dental radiographs. The aim of the present study was to evaluate the influence of four different ambient light conditions on the depiction ability of small contrast elements in digital dental images. By means of an exactly known test object, a large number of observers, and a receiver operating characteristics (ROC) analysis, the influence of ambient light on observer performance should be investigated.

\section{Materials and methods}

\section{Test object}

An aluminum step wedge $(40 \times 30 \mathrm{~cm})$ with eight steps of increasing thickness $(3-10 \mathrm{~mm})$ containing 32 boreholes (diameter, $1 \mathrm{~mm}$; depth, 0.05, 0.10, 0.30, and $0.50 \mathrm{~mm}$ ) was used as described in a previous study [7]. Each step was divided into five equal segments (length $6 \mathrm{~mm}$; width, $5 \mathrm{~mm}$ ), four of which were provided with one borehole (Fig. 1). The localization of each hole was randomized avoiding a close relation to the border zones of each segment. Based on $\mathrm{CNC}$ device and a plain drilling machine, the holes were burred with a precision of $0.01 \mathrm{~mm}$ in length and width (Chiron-Werke GmbH \& Co. KG, Tuttlingen, Germany). Thus an equal thickness of aluminum inside the boreholes could be realized.

Image acquisition

A direct digital cephalometric unit (Orthophos DS Ceph, Sirona Dental System GmbH, Bensheim, Germany) equipped with a CCD sensor with an effective detector array of $138.6 \times$ $5.9 \mathrm{~mm}^{2}$ was used for image acquisition, providing a spatial resolution of $282 \times 282 \mathrm{dpi}$. The wedge phantom was

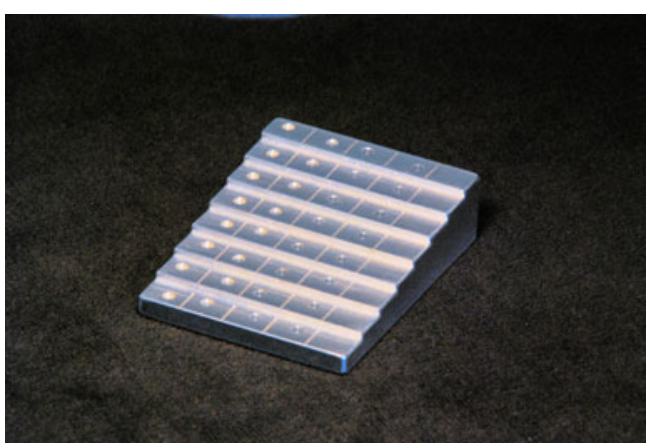

Fig. 1 Aluminum step wedge with boreholes

positioned in such a way that its back (and front) side was aligned parallel to the receptor at a distance of $26 \mathrm{~mm}$ as described in a previous study [8]. Radiographs were produced over the entire exposure range and the image exposing the maximum contrast as assessed from the extreme grayscale at the thick and the thin end of the wedge was selected (Photopaint 11.0, Corel Corporation, Ottawa, Ontario, Canada) for further evaluation. The mean gray value for each segment was additionally determined using the identical software.

\section{Image processing}

The original radiograph was exported as uncompressed 8-bit tiff file and subsequently separated into its single cells images by the image editing software specified above, resulting in 40 single images with a spatial resolution of $282 \mathrm{dpi}^{2}$ each. Thirty-two images comprised a small contrast feature in terms of a borehole; eight images were without. In order to obtain a 1:1 numeric distribution between images with and without borehole, each of the eight images without borehole was represented four times. This resulted in a total of 64 images (32 with borehole, 32 without). For display on monitor, each single image was presented as uncompressed tiff file using a black background.

\section{Image evaluation}

Images were presented on a 17-in CRT monitor (XTA 3813 MT, Iiyama Electric Co. Ltd., Nagano, Japan; resolution: $1,024 \times 768$ pixels, true color, blacktest positive, $170 \mathrm{~cd} / \mathrm{m}^{2}$ ). The monitor represented the standard at the time of study begin and was quality tested according to German regulations for dental radiographic viewing monitors both on a daily and monthly basis. The eye-to-screen distance was between 60 and $80 \mathrm{~cm}$ and the angle for viewing around $35^{\circ}$. Different background illuminations of $0 \mathrm{~lx}$ (completely dark), $50 \mathrm{~lx}$ (corresponding to 50 candles at $1 \mathrm{ft}$ distance), $200 \mathrm{~lx}$ (corresponding to the illumination of floors), and $500 \mathrm{~lx}$ (bright illumination in offices/receptions) were created by dimming an indirect halogen light (floor uplighter, positioned $2 \mathrm{~m}$ in front 
of the monitor, illuminating the ceiling with approximately $45^{\circ}$ ) manually. Lux represent a standardized unit to express the perception of brightness for human and is equal to 1 lumen per $\mathrm{m}^{2}$. The background illumination was assessed with a lux meter (Luxmeter LM-1010, ELVOS GmbH, Ludwigsburg, Germany) in the middle of the monitor which was turned off during evaluation. The time for adapting the eye to the different background illumination was at least $10 \mathrm{~min}$ for each observer.

Twenty observer (students of the Dental School in their final year) stated their blinded decision whether or not they could perceive a dark spot representing a hole using the following five-point confidence scale: 1 = "hole definitely visible", 2 = "hole probably visible", 3 = "uncertain, whether or not a hole is visible", 4 = "hole probably not visible," and 5 = "hole definitely not visible." The order of images shown was randomized with respect to the particular background illumination. All observers were briefed and calibrated on the procedure prior to participation by showing them one sample image with and without a borehole. There were no time limits for evaluation. All images were viewed subsequently at different background illumination in one session of an appropriate duration of $20 \mathrm{~min}$. Windowing and leveling was not allowed for the observers. The ratings were recorded by one author (T. S.) and transferred into spreadsheet software (Microsoft Excel 2003, Microsoft Corporation, Redmond, USA) for statistical evaluation. The evaluation was repeated under randomization after a time interval of at least 30 days, thus resulting in total of 10,240 readings (64 images $\times 4$ background illuminations $\times 20$ observer $\times 2$ observations).

\section{Statistical data evaluation}

For each observer and background illumination, the respective ROC was calculated, with the threshold rating assigned to the negative proportion of observations. The area beneath each curve $(\mathrm{Az})$ was estimated nonparametrically. Accordingly, sensitivity (SN) and specificity (SP) were obtained. Az values were compared between the different background illuminations using the nonparametric Friedman test to determine statistically significant differences between the four background illuminations. The level of significance was set at $p<0.05$.

The data were averaged over all observers and both observations and the intra-rater reliability was estimated using the

Table 1 Mean sensitivity (SN), specificity (SP), and areas beneath the curves $(\mathrm{Az})$ for the different background illuminations (in lux)

\begin{tabular}{llll}
\hline Ambient light & SN & SP & Az \\
\hline 0 & 0.58 & 0.7 & 0.735 \\
50 & 0.53 & 0.72 & 0.728 \\
200 & 0.5 & 0.74 & 0.735 \\
500 & 0.53 & 0.73 & 0.788 \\
\hline
\end{tabular}

Table 2 Intraclass correlation coefficient (ICC) and $95 \%$ confidence interval showing the readers reliability at different background illuminations (in lux)

\begin{tabular}{ll}
\hline Ambient light & ICC \\
\hline 0 & $0.677(0.758)$ \\
50 & $0.706(0.781)$ \\
200 & $0.683(0.762)$ \\
500 & $0.737(0.806)$ \\
\hline
\end{tabular}

intraclass correlation coefficient (ICC). Additionally, the SN and SP were determined for the main background grayscale and differences between the four background illuminations were evaluated separately.

\section{Results}

SN averaged over all observers and both observations was the highest for $0 \mathrm{~lx}$ and the lowest for $200 \mathrm{~lx}$ background illumination (Table 1). SP was the highest for $200 \mathrm{~lx}$ and the lowest for $0 \mathrm{~lx}$ background illumination (Table 1). The differences between the four background illuminations were marginal for SN and SP (Table 1). Accordingly, Az values showed only slight variance between the four viewing conditions (Table 1) without significant difference $(p=0.796)$.

The intra-rater reliability, i.e., concordance between first and second observation, was highest for the viewing conditions at $500 \mathrm{~lx}$ and the lowest for $0 \mathrm{~lx}$ (Table 2). The highest numbers of positive (correct) results (evaluations)

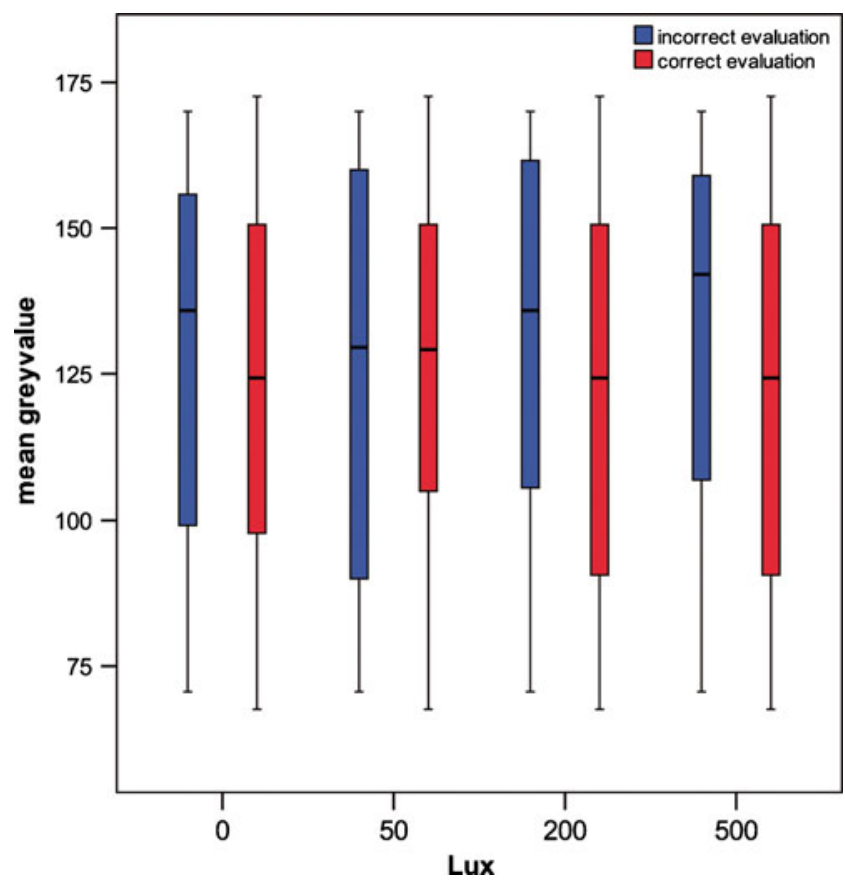

Fig. 2 Boxplots showing correct evaluations (red) and incorrect evaluations (blue) according to the mean gray value of the images and background illumination. At 200 and 500 lx, fewer incorrect evaluations were observed in low gray value images than at 0 and $50 \mathrm{~lx}$ 
were obtained at a background illumination of 200 and $500 \mathrm{~lx}$, at brighter mean grayscale of the images (Fig. 2). The differences were not statistically significant.

\section{Discussion}

The digitization of dental radiology in dental offices is associated with completely different viewing conditions when compared to conventional radiography. There is only scarce knowledge on the effect of ambient light on the diagnostic performance in digital dental radiology. However, knowledge on this is important for the daily practice since ambient light generally is known to be very variable between different dental offices.

The present study showed that within a range of 0 $500 \mathrm{~lx}$, the diagnostic performance in terms of sensitivity and specificity was not significantly influenced by the different background illuminations. Pollard et al. evaluated the influence of ambient light of $50 \mathrm{~lx}$ when compared to $0 \mathrm{~lx}$ in chest radiology for the detection of suspicious nodule, and in agreement to our findings, they found no statistically significant differences in sensitivity and specificity for slightly elevated background illuminations [5]. It could additionally be shown that a moderate background illumination of $<50 \mathrm{~lx}$ may even improve the observers performance when LCD monitors are used [3]. Chawla and Samei concluded a background illumination of 75-150 lx range to be an ideal setup for typical LCD's [4]. The good diagnostic performance at slightly elevated background illumination may be explained by the low diffuse reflection coefficients and high luminance ratio of LCD monitors [4]. The background illumination in the present study included elevated ambient light levels of 200 and 500 lx. These were used according to recommendations of the DIN-norm 6868-57. In these a background, illumination between 300 and $500 \mathrm{~lx}$ is recommended for diagnostic of radiographs exposed on monitors [9]. Interestingly when evaluating the diagnostic performance according to the mean gray value of the images, the study showed for elevated background illuminations of 200 and $500 \mathrm{~lx}$ less diagnostic errors in lower gray images than for 0 and $50 \mathrm{~lx}$. This seems to be in contrast to studies which showed that values above $450 \mathrm{~lx}$ reduce perception of micronodules and pulmonary lines in chest image on cathode ray tubes $[10,11]$. A recent study could show that the detection of caries lesions at a background illumination of $1,000 \mathrm{~lx}$ is significantly inferior to the detection at $50 \mathrm{~lx}$ background illumination on a LCD monitor [12]. It is difficult to compare the results since it is unknown to what extend the different types of monitors, e.g., TFT, LCD or CRT, as used in our study may be influenced by the ambient background illumination with regard to the resulting diagnostic performance. However, several studies proved elevated diagnostic performance in the detection of lung nodules for LCD when compared to CRT as used in the present study [13]. This is mainly related to the lower luminance ratio of CRT [13]. Nevertheless, it seems that CRT is superior in low-attenuation areas of thorax radiographs, whereas LCD may be advantageous in high-attenuation areas [14].

Contrary to the clinical approach of all cited studies, we based our evaluation on experimental radiographs. These included small contrast features which had to be detected by the viewer. The use of phantoms allows clear determination of the sensitivity and specificity of a diagnostic system, and results are not depending on individual interpretation [15]. The rather low sensitivity values indicate that the diagnostic task, i.e., perception of small round-shaped dark spots at varying locations within an image of gray was not as simple as suggested. Finally and within the limits of a phantom-based study, the data indicate that the impact of background illumination seems to be moderate within the range of 0-500 $\mathrm{lx}$ for detecting small contrast features in digital dental images displayed on CRT monitors. With regard to the different monitor devices and potential impact on diagnostic performance, we suggest a clinical study design to confirm the findings of the present study. A comparison between CRT and LCD for the diagnostic performance of digital dental radiographs could additionally reveal important information on optimum monitor display of digital dental radiographs.

Conflict of interest The authors declare that they have no conflict of interests.

\section{References}

1. Geissler O, Rother U (2007) The film-based printout using tetenal printing solution II. Int J Comput Dent 10:285-291

2. Wenzel A (1999) Matters to consider when implementing direct digital radiography in the dental office. Int J Comput Dent 2:269-290

3. Pollard B, Chawla AS, Delong DM, Hashimoto N, Samei E (2008) Object detectability at increased ambient lighting conditions. Med Phys 35:2204-2213

4. Chawla AS, Samei E (2007) Ambient illumination revisited: a new adaptation-based approach for optimizing medical imaging reading environments. Med Phys 34:81-90

5. Pollard BJ, Samei E, Chawla AS, Beam C, Heyneman LE, Hurwitz-Koweek LM et al (2012) The effect of ambient lighting in chest radiology reading rooms. J Digit Imaging. doi:10.1007/ s10278-012-9459-5

6. Siegel EL (1998) Economic and clinical impact of filmless operation in a multifacility environment. J Digit Imaging 11:42-47

7. Grassl U, Schulze RKW (2007) In vitro perception of low-contrast features in digital, film, and digitized dental radiographs: a receiver operating characteristic analysis. Oral Surg Oral Med Oral Pathol Oral Radiol Endod 103:694-701

8. Kühl S, Krummenauer F, Dagassan-Berndt D, Lambrecht JT, d'Hoedt B, Schulze RKW (2011) Ink-jet printout of radiographs 
on transparent film and glossy paper versus monitor display: an ROC analysis. Clin Oral Investig 15:351-356

9. Schöfer H (2001) Monitorbefundung in Tageslicht beleuchteter Umgebung basierend auf DIN 6868-57. Bayerisches Landesamt für Arbeitsschutz, Arbeitsmedizin und Sicherheitstechnik

10. Scharitzer M, Prokop M, Weber M, Fuchsjager M, Oschatz E, Schaefer-Prokop E (2005) Detectability of catheters on bedside chest radiographs: comparison between liquid crystal display and highresolution cathode-ray tube monitors. Radiology 234:611-616

11. Goo JM, Choi JY, Im JG, Lee HJ, Chung MJ, Han D et al (2004) Effect of monitor luminance and ambient light on observer performance in soft-copy reading of digital chest radiographs. Radiology 232:762-766
12. Hellén-Halme K, Lith A (2012) Effect of ambient light level at the monitor surface on digital radiographic evaluation of approximal carious lesions: an in vitro study. Dentomaxillofac Radiol 41:192-196

13. Buls N, Shabana W, Verbeek P, Pevenage P, De Mey J (2007) Influence of display quality on radiologist' performance in the detection of lung nodules on radiographs. Br J Radiol 80:738-743

14. Balassy C, Prokop M, Weber M, Sailer J, Herold C, SchaeferProkop C (2005) Flat-panel display (LCD) versus high-resolution grays-scale display (CRT) for chest radiography: an observer preference study. Am J Roentgenol 184:752-756

15. Otis L, Sherman RG (2005) Assessing the accuracy of caries diagnosis via radiograph. Film versus print. J Am Dent Assoc 136:323-330 